\title{
Risk analysis of treatment of unruptured aneurysms
}

\author{
P Mitchell, J Jakubowski
}

\begin{abstract}
Objectives-To calculate the expected number of life-years saved by surgical treatment of unruptured intracranial aneurysms at ages over 20 years.

Methods-An actuarial risk analysis of the treatment of unruptured intracranial aneurysms based on data from the International Study of Unruptured Intracranial Aneurysms (ISUIA). The benefits of operative treatment are calculated in terms of average life-years saved.

Results-Results are presented as graphs of life-years saved or lost against age at the time of operative treatment for three groups of aneurysms: those under $10 \mathrm{~mm}$ in diameter with no history of subarachnoid haemorrhage (SAH) from another aneurysm, those under $10 \mathrm{~mm}$ in diameter and a history of previous SAH from a different aneurysm, and those over $10 \mathrm{~mm}$ in diameter. Life-years are lost at all ages in the group under $10 \mathrm{~mm}$ with no history of SAH. For the group under $10 \mathrm{~mm}$ with a history of SAH about 4 years are saved at age 20 declining to 0 at around age 50 . For aneurysms $10 \mathrm{~mm}$ or more in diameter about 8 life-years are saved at age 20 declining to 0 at around 50 .

Conclusions-Clipping of unruptured aneurysms under $10 \mathrm{~mm}$ in diameter with no history of subarachnoid haemorrhage is not justified on actuarial grounds. Intervention in other unruptured aneurysms produces benefits in life expectancy up to the age of 50. There may be subgroups of aneurysms in which larger benefits exist. (F Neurol Neurosurg Psychiatry 2000;68:577-580)
\end{abstract}

Keywords: intracranial aneurysms; risk analysis

This analysis is based on the results of the International Study of Unruptured Intracranial Aneurysms (ISUIA). ${ }^{1}$ The ISUIA data gives the best available assessment of the risks posed to a patient by an unruptured intracranial aneurysm and the risks of operative treatment. Craniotomy and clipping of aneurysms is the conventional operative treatment. In recent years it has become possible to occlude intracranial aneurysms with metal coils by an endovascular route. Both clipped and coiled cases are included in the prospective operative arm of the ISUIA. Before the publication of the ISUIA data several studies had addressed the decision of whether of not to surgically treat unruptured intracranial aneurysms. ${ }^{2-8} \mathrm{~A}$ total of about 3500 patient-years of follow up had been published for patients with untreated unruptured aneurysms. Risk analyses based on this data ${ }^{2}{ }^{3}$ suggested substantial benefits from surgical treatment.

The ISUIA included 12023 patients years of follow up of untreated unruptured aneurysms and 1172 operated unruptured aneurysms. The results showed a higher surgical complication rate from treatment and a lower annual aneurysm rupture rate than was apparent from earlier data. The benefits of treatment therefore seem more modest than was previously thought.

\section{Method}

The mathematical strategy used was to derive an age specific annual risk of death from the 1994 mortality statistics of the total population of England and Wales, and to add to it an estimate of the risk posed by an unruptured aneurysm. This total annual risk was used to calculate the remaining life expectancy of persons harbouring untreated unruptured aneurysms. The remaining life expectancy of those undergoing surgery was calculated by taking the life expectancy of the normal population and adjusting it to allow for the operative risks. The result was compared with the untreated case to calculate average life-years saved or lost by operating. A more detailed description of the mathematical method is given in the appendix.

In the group of aneurysms under $10 \mathrm{~mm}$ in diameter with no history of SAH the ISUIA found one haemorrhage in about 3500 patientyears of follow up. This gives a bleed rate of $0.028 \%$ per patient-year. Because of the uncertainty resulting from only a single observed bleed the authors gave the rate as "under $0.05 \%$ per patient year". The figure of $0.05 \%$ per year was used here. The bleed rate used for aneurysms under $10 \mathrm{~mm}$ in diameter with a history of SAH from another source was $0.55 \%$ and that used for aneurysms $10 \mathrm{~mm}$ or more in diameter was $0.88 \%$ per patient year. The ISUIA split aneurysms of $10 \mathrm{~mm}$ or more in diameter into groups with and without a history of SAH but there was no significant difference between the bleed rates of the two groups so we have combined them to yield a bleed rate of $0.88 \%$ per patient year.

The annual risk posed by an aneurysm was estimated by multiplying the bleed rate given in the ISUIA report by the "bad outcome rate" after SAH. Reported series and previous risk analysis have divided outcomes after subarachnoid haemorrhage into death or serious disability and full recovery or mild disability. That convention is followed. Serious disability is usually classified as requiring assistance with the acts of daily living. A modified Rankin score 


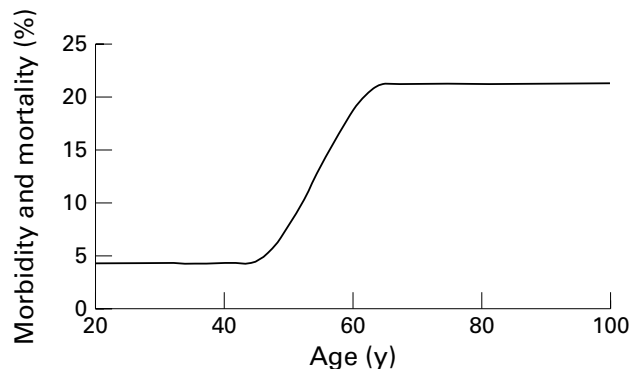

Figure 1 The age dependent function used to model operative morbidity and mortality. The morbidity included are all cases requiring help with acts of daily living 1 year after surgery. The function gives the graphs of figure 3 their sigmoid form. This is only one among many functions which could have been chosen with equal validity.

of 3-5 was used by ISUIA and here. Death was the result of $66 \%$ of haemorrhages in the ISUIA study. The morbidity rate was not given but other studies of outcome after subarachnoid haemorrhage suggest that $30 \%$ of the survivors make a "poor" neurological recovery. ${ }^{9-11}$ Combining these figures leads to an overall bad outcome rate after rupture of an aneurysm under follow up of $76 \%$. The bleed risk is scaled by this factor before adding it to the population's age specific mortality to obtain the overall risk.

The morbidity and mortality for clipping and coiling unruptured aneurysms is calculated in the ISUIA from 1172 cases. Two factors complicate the division of outcome into convenient "good" and "bad" groups.

Firstly, the operative complication rate increases with increasing age. The results of the ISUIA give rates in the age ranges: under 45 years ( $4.3 \%$ poor outcomes as defined above), 45 to 64 years (9.6\% poor outcomes), and 65 or over (21.3\% poor outcomes). A sigmoid spline function has been used to fit this age stratification for the present study (fig 1).

Secondly the ratio of morbidity to mortality is different for the haemorrhagic group and the operative group. Of those with a bad outcome after a subarachnoid haemorrhage, about $86 \%$ will be dead whereas for of those with a bad outcome after operative treatment, only about $25 \%$ will be dead. This complicates the task of selecting comparable groups. The compromise we use is to count those requiring care every day or more often (modified Rankin 3-5) 1 year after the operation as bad outcomes and the rest as good.

Because operative outcomes may vary between units and between coiling and clipping and may improve in the future we have included graphs showing the effects of different operative risks on the three aneurysm groups (fig 2).

Results

Our results are summarised in figs 2,3 , and 4 . Figure 3 shows the life expectancy curves for the normal population, the operated group, and the conservatively managed group for aneurysms $10 \mathrm{~mm}$ or more in diameter and is included to illustrate the approach taken to the calculations. Figure 4 shows life-years saved against age for the three aneurysm groups:
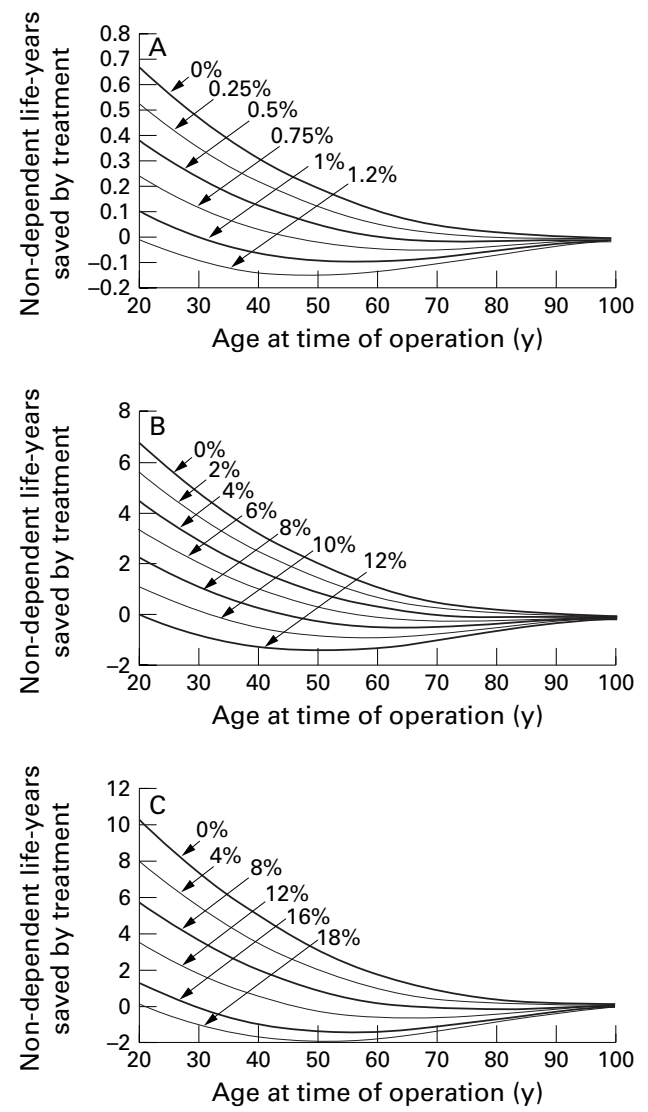

Figure 2 Illustrations of the model's sensitivity to operative risks; the figures refer to combined morbidity and mortality. The graphs show expected non-dependent life-years saved against age at time of treatment for aneurysms under $10 \mathrm{~mm}$ in diameter with no history of $S A H(A)$, under $10 \mathrm{~mm}$ with such a history $(B)$, and over $10 \mathrm{~mm}(C)$. Several operative risks are spaced between $0 \%$ and the lowest figure to give no benefit at any age in such aneurysms.

those under $10 \mathrm{~mm}$ with no history of SAH, those under $10 \mathrm{~mm}$ with a history of $\mathrm{SAH}$, and those $10 \mathrm{~mm}$ or more. The graphs in figure 4 are sigmoid because of the function we have used to fit the age stratified operative outcome data (fig 1). This function is to some extent arbitrary. We use it because it is continuous and does not go lower than the minimum reported operative risk or higher than the maximum. Many other functions, both continuous and discontinuous, could have been used. We have tried several and have found that the points at which the lines cross 0 are highly insensitive to the function used (as long as it agrees with the ISUIA data). The safest group of aneurysms are those under $10 \mathrm{~mm}$ in diameter in persons with no history of SAH from other sources and in this group operating at any age reduced life expectancy. The other two groups showed a reduction in life expectancy from the age of about 50 onwards and modest gains in life expectancy for operating at younger ages. The maximum gains were at the youngest age and amounted to 4 life-years in the smaller aneurysms with a history of SAH and 8 life-years in the larger aneurysms. We have also calculated the lowest operative risks which results in no saving in life-years at any age. This risk was $1.2 \%$ for the small aneurysms with no 


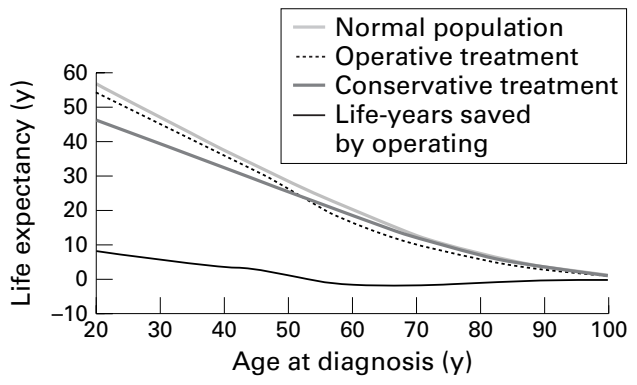

Figure 3 Life expectancy against age for the normal population of England and Wales based on 1994 census age specific mortality, for those harbouring unruptured aneurysms larger than $10 \mathrm{~mm}$ in diameter and for those undergoing operative treatment of unruptured aneurysms. The greater than $10 \mathrm{~mm}$ group is chosen for illustrative purposes.

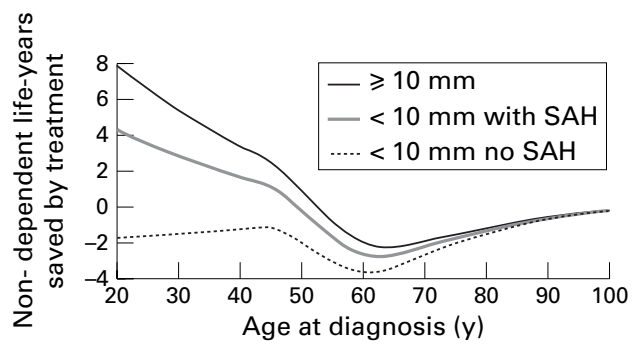

Figure 4 Average non-dependent life-years saved or lost by clipping unruptured aneurysms in the three groups.

history of SAH, $12 \%$ for small aneurysms with a history of $\mathrm{SAH}$, and $18 \%$ for larger aneurysms. Figure 2 shows a spread of operative risks between these values and 0 .

\section{Discussion}

Previous risk analyses have suggested a substantial benefit in life-years gained from craniotomy and clipping of unruptured aneurysms. These results were based on earlier data concerning the risks of unruptured aneurysms and the risks of surgery. Ideas about the risks of unruptured aneurysms are derived from two types of data. The most direct data are from follow up studies of unruptured, untreated aneurysms. When pooled, such studies contained about 3500 patient-years of follow up before ISUIA. They suggested that the bleed risk from this group was around $1 \%$ to $2 \%$ a year. Around 835 life-years of follow up of aneurysms in the under $10 \mathrm{~mm}$, no history of $\mathrm{SAH}$ group can be identified among these and in this subgroup the risk is indeed small $(0.24 \%$ haemorrhages a year) but still larger than the ISUIA result $(0.05 \%)$. The reason for the difference in the bleed rates found by previous studies and the ISUIA remains enigmatic but the ISUIA numbers are far larger than are all previous data put together.

The other principle source of annual bleed risks is via the indirect calculation of dividing the incidence of SAH by the prevalence of aneurysms (from angiographic or postmortem data) within a population. This calculation yields a bleed rate of around $1 \%$ a year for all the three aneurysm subtypes considered in ISUIA. This method carries some weight because the numbers involved in incidence and prevalence studies are large and because the figures do not vary greatly from one western population to another. Why then does this figure disagree by a factor of more than 20 with the ISUIA data on the safest group? One possible explanation is that an assumption which underlies the calculation is faulty. This assumption is that the risk of haemorrhage from an aneurysm does not change with time. The paradox would be solved if aneurysms went through a period of high risk just after formation and then stabilised to a low risk. This scenario was proposed by Wiebers et al in $1986^{12}$ to account for this and another paradoxical finding. These authors noted that most aneurysms which present with subarachnoid haemorrhage are under $10 \mathrm{~mm}$ in diameter whereas most unruptured aneurysms which bleed under follow up are greater than $10 \mathrm{~mm}$ in diameter and the discrepancy is too great to be explained on the grounds of small aneurysms being more common.

Our calculations are based on three statistics: the risks of unruptured aneurysms, the poor outcome rate of operations, and the life expectancy of the normal population. None of these values are known exactly. Considering first the life expectancy of the normal population this is fairly accurately known now, but will it be longer for those now in their 30 s and 40 s than for those in later years on which our estimates are largely based?

Turning to the bleed risk of unruptured aneurysms, there may be subgroups of aneurysms which pose a particularly high risk or are particularly safe to treat and may show greater benefits than the generally modest ones that we have calculated. Familial aneurysms or basilar tip aneurysms are possible contenders and may merit further study. Another factor yet to be fully considered is the efficacy of medical treatment. Slosberg has found a very low rupture rate from previously ruptured aneurysms treated with careful hypotensive regimes. ${ }^{13} 14$ Moreover, many of his patients were treated conservatively because they were considered to pose an unacceptably high surgical risk.

Turning finally to operative outcome data, the ISUIA data on operative outcomes was collected from multiple centres, the numbers involved were large, and the operative outcome data were collected prospectively with independent assessment. For these reasons we consider that the ISUIA outcome dataset is the most reliable available at the present time but of all the statistics used in this study this is probably the most liable to future revision. Greater note was taken of cognitive impairment in the ISUIA than previously and this largely accounts for the results being poorer than in some other series. Even so some surgeons have published more favourable operative results ${ }^{15-18}$ and the performance of coiling is as yet unknown. A further problem is that the function we have chosen to represent the changing operative risk with age is a convenient estimate only and cannot be exactly correct.

With these uncertainties an analysis of confidence intervals would be helpful but it is not known how measurements of bleed rates and operative risks are distributed around the true 
value so meaningful $95 \%$ confidence intervals cannot be offered. The values we have used remain the best estimates available.

\section{Conclusions}

Based on the results of the ISUIA the demographic impact of operative treatment of unruptured aneurysms varies from a modest benefit to a slight detriment. In those patients with unruptured aneurysms under $10 \mathrm{~mm}$ in diameter and no history of SAH from another source life-years are lost on average by operative treatment. Patients with other unruptured aneurysms show a modest benefit from operative treatment up to the age of 50 . There may be subgroups of aneurysms in which operative treatment carries greater benefits but this will only be known when the risks posed by such aneurysms and the risks of treatment are known. Risk analysis is only one aspect of the complex problem of unruptured aneurysms. The average number of life-years saved is an abstraction which most patients will have difficulty applying to themselves. The final decision to operate or not must be guided by clinical judgement and the patients' wishes.

\section{Appendix: mathematical method}

The annual risk posed by an unruptured aneurysm $(r)$ was taken as the bleed rate multiplied by the bad outcome rate for $\mathrm{SAH}$ as discussed above. Over the age of 20, the age specific mortality of human populations is known to be well approximated by an exponential function of the form $e^{-a+b x}$ where $a$ and $b$ are constants and $x$ is age in years. ${ }^{19} 20$ Using least squares regression to fit this to the age specific moralities for England and Wales in $1994^{21}$ yields:

$$
m=e^{-9.958+0.0903 x}
$$

(This method was used in the present context by Chang and Kirino ${ }^{2}$.) This function was used as an age specific annual risk of death for the normal population and $r$ was added to it to give a total annual risk $M$ for those harbouring unruptured aneurysms $(M=m+r)$. Survival to age $x$ (which will be called $S(x)$ ) after diagnosis at age $a$, is then calculated using the recurrence relation: $S(x)=(1-M(x)) S(x-1), S(a)$ being 1 . The lowest age considered is 20 years for two reasons: subarachnoid haemorrhage is rare below this age so the period up to 20 years has limited relevance, and the exponential function is a good fit for observed death rates only above 20 years of age. Remaining life expectancy at age a is then given by:

$\int_{\mathrm{a}}^{\infty} S(x) d t$
Using this method, curves of life expectancy against age can be derived from the starting point of annual death rates. The life expectancy of a patient with an aneurysm that has been clipped successfully is assumed to be normal. The operative morbidity and mortality are included by assuming an initial survival of $\left(1-r_{o}\right)$ where $r_{o}$ is the operative risk (of a bad outcome). An age dependent sigmoid function is used for $r_{0}$ (fig 1). All calculations were done numerically with the program Matlab 5.1 running on a PC. The quadrature method of integration was used.

1 Anonymous. Unruptured intracranial aneurysms: risk of rupture and risks of surgical intervention. International Study of Unruptured Intracranial Aneurysms Investigators. Study of Unruptured Intracranial Any

2 Chang HS, Kirino T. Quantification of operative benefit for unruptured cerebral aneurysms: a theoretical approach. $\mathcal{F}$ Neurosurg 1995;83:413-20.

3 Leblanc R, Worsley KJ. Surgery of unruptured, asymptomatic aneurysms: a decision analysis. Can $\mathcal{f}$ Neurol Sci 1995;22:30-5

4 Leblanc R, Worsley $\mathrm{KJ}$, Melanson $\mathrm{D}$, et al. Angiographic screening and elective surgery of familial cerebral aneurysms: a decision analysis. Neurosurgery 1994;35:9-19.

5 Rinkel GJ, Djibuti M, van Gijn J. Prevalence and risk of rupture of intracranial aneurysms: a systematic review. Stroke 1998;29:251-6.

6 Rosenorn J, Eskesen V. Does a safe size-limit exist for unruptured intracranial aneurysms? Acta Neurochir (Wien) 1993;121:113-18.

7 ter Berg HW, Dippel DW, Habbema JD, et al. Treatment of intact familial intracranial aneurysms: a decision-analytical intact familial intracranial aneurysms: a decis
approach. Neurosurgery 1988;23:329-34.

8 van Crevel H, Habbema JD, Braakman R. Decision analysis of the management of incidental intracranial saccular aneurysms. Neurology 1986;36:1335-9.

9 Deruty R, Patet JD, Mottolese C, et al. Long-term outcome of the management of ruptured intracranial aneurysm. Review of 328 consecutive patients treated over a period of 12 years. Neurological Research 1988;10:217-20.

10 Ljunggren B, Fodstad H, von Essen C, et al. Aneurysmal subarachnoid haemorrhage: overall outcome and incidence of early recurrent haemorrhage despite a policy of acute stage operation. Br $\mathcal{F}$ Neurosurg 1988;2:49-53.

11 Saveland H, Hillman J, Brandt L, et al. Overall outcome in aneurysmal subarachnoid hemorrhage. A prospective study from neurosurgical units in Sweden during a 1-year period. from neurosurg 1992;76:729-34.

12 Wiebers DO, Whisnant JP, Sundt TM Jr, et al. The significance of unruptured intracranial saccular aneurysms. $f$

13 Slosberg PS. Unexpected results in long-term medically treated ruptured intracranial aneurysm including data on 14 patients followed more than 30 years each. Acta Neurochir (Wien) 1997;139:697-705.

14 Slosberg PS. Zero percent mortality due to recurrent hemorrhage in follow-up of medically treated ruptured single intracranial aneurysms: a 23-year study. Transactions of the American Neurological Association 1979;104:180-3.

15 Yoshimoto T, Mizoi K. Importance of management of unruptured cerebral aneurysms. Surg Neurol 1997;47:522-6.

16 Mizoi K, Yoshimoto T, Nagamine Y, et al. How to treat incidental cerebral aneurysms: a review of 139 consecutive dental cerebral aneurysms: a review

17 Solomon RA, Fink ME, Pile-Spellman J. Surgical management of unruptured intracranial aneurysms. $\mathcal{F}$ Neurosurg ment of unrupture

18 Rice BJ, Peerless SJ, Drake CG. Surgical treatment of unruptured aneurysms of the posterior circulation. $f \mathrm{Neu}$ rosurg 1990;73:165-73.

19 Beck JR, Pauker SG, Gottlieb JE, et al. A convenient approximation of life expectancy (the "DEALE"). II. Use in medical decision-making. Am f Med 1982;73:889-97.

20 Beck JR, Kassirer JP, Pauker SG. A convenient approximation of life expectancy (the "DEALE"). I. Validation of the method. Am f Med 1982;73:883-8.

21 McGuigan S. Office of Health Economics compendium of health statistics 1997. London: OHE, The Stationary office, 1997. 